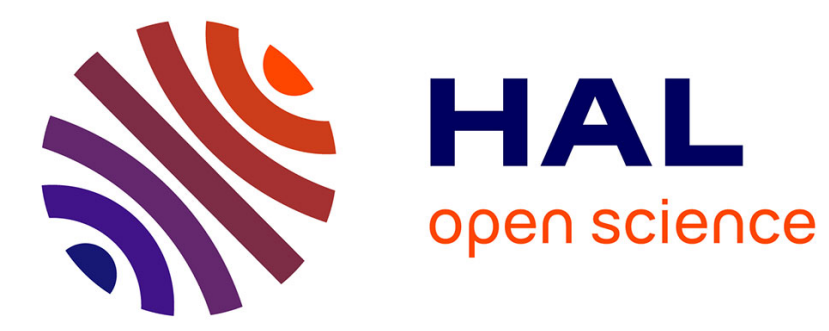

\title{
Les musées d'histoire et de la littérature soviétique face à la perestroïka
}

Sofia Tchouikina

\section{To cite this version:}

Sofia Tchouikina. Les musées d'histoire et de la littérature soviétique face à la perestroïka. Revue d'Etudes Comparatives Est-Ouest, 2011, 42 (3), pp.89-114. halshs-01904554

\section{HAL Id: halshs-01904554 \\ https://shs.hal.science/halshs-01904554}

Submitted on 26 Oct 2018

HAL is a multi-disciplinary open access archive for the deposit and dissemination of scientific research documents, whether they are published or not. The documents may come from teaching and research institutions in France or abroad, or from public or private research centers.
L'archive ouverte pluridisciplinaire HAL, est destinée au dépôt et à la diffusion de documents scientifiques de niveau recherche, publiés ou non, émanant des établissements d'enseignement et de recherche français ou étrangers, des laboratoires publics ou privés. 


\title{
LES MUSÉES D'HISTOIRE ET DE LA LITTÉRATURE SOVIÉTIQUE FACE À LA PERESTROÏKA
}

\author{
SOFIA TCHOUIKINA
}

Résumé : Pendant la perestroïka, le fonctionnement des musées d'histoire et de la littérature soviétiques a été critiqué par les médias et par l'intelligentsia. L'article étudie l'impact de la libéralisation de la vie politique et économique, de l'abolition de la censure, de la crise budgétaire et de l'ouverture à l'international sur ces établissements. Forcés de se reconvertir pour attirer les visiteurs, ils ont modernisé leurs salles d'expositions; les relations avec le public sont devenues leur préoccupation principale. Ces anciennes institutions idéologiques ont délaissé les interprétations macro-historiques totalisantes au profit des détails et ont mis l'accent sur la vie quotidienne, les vies privées et l'histoire locale.

Mots-cLÉs : Russie, perestroïka, musées d'histoire, musées littéraires, mémoire collective. 


\section{INTRODUCTION}

Dans la mémoire des responsables des musées d'histoire et de la littérature soviétique, la perestroïka apparaît comme un moment de grand tournant dans leur trajectoire professionnelle. D'après les témoins de cette époque mouvementée, qui exercent aujourd'hui des fonctions importantes au sein des mêmes institutions, ils furent confrontés à l'hostilité des partisans des réformes et durent élaborer des stratégies de reconversion afin d'acquérir une nouvelle raison d'être dans une société transformée. Certains musées ont disparu dans la tourmente mais la plupart ont poursuivi leurs activités, parfois en changeant de nom, tandis que de nouveaux musées, consacrés à la résistance au régime et à la culture non officielle, étaient fondés.

L'interprétation de l'histoire soviétique préoccupe désormais la société russe qui ne trouve pas de solutions satisfaisantes, à part le « patriotisme de désespoir »(Oushakine, 2009 ; Smith, 2002). Le fonctionnement de toutes les institutions responsables de la transmission de la mémoire historique aux nouvelles générations (les écoles, les universités, les manuels, les archives, l'industrie cinématographique, les maisons d'éditions) est systématiquement mis en cause. Pourtant, l'analyse sociologique approfondie du fonctionnement de ces institutions reste à faire. L'échantillon d'entretiens avec les responsables des musées qui évoquent leur expérience professionnelle, met en lumière des logiques, biographiques et institutionnelles, d'interprétation de l'histoire soviétique par ces établissements.

Quinze entretiens avec des directeurs, des vice-directeurs ou des collaborateurs scientifiques de haut niveau des musées d'histoire et de la littérature soviétique, conduits dans les années 2000 à Saint-Pétersbourg, à Nijni-Novgorod et dans d'autres villes, constituent la source principale de cet article ${ }^{1}$. Ils sont complétés par des entretiens avec des responsables d'autres institutions culturelles publiques, des représentants de fondations, des spécialistes de la politique culturelle. Les publications des muséologues sur l'état des lieux de la profession et les articles de presse ont également servi de contexte pour l'interprétation des entretiens.

\footnotetext{
1. Musée de l'Histoire politique de la Russie (Saint-Pétersbourg, 1999, 2000), musée Smolny (Saint-Pétersbourg, 2000), musée d'Histoire de Saint-Pétersbourg (Saint-Pétersbourg, 2000), musée de la Défense de Leningrad (Saint-Pétersbourg, 2000), musée Anna-Akhmatova à Fontannyj Dom (Saint-Pétersbourg, 2001, 2004, 2008), musée-appartement de Mikhaïl Zochtchenko (Saint-Pétersbourg, 2010), musée de Vladimir Nabokov (Saint-Pétersbourg, 2000), musée-appartement des Elizarov (Saint-Pétersbourg, 2000), musée de l'Histoire de la Religion (Saint-Pétersbourg, 2008), musée Andreï-Sakharov (Nijni-Novgorod, 2004), musée Maxime-Gorki (Nijni-Novgorod, 2004), musée National de la République d'Oudmourtie (Iževsk, 2004), musée d'Histoire locale (Kirov/Vyatka, 2004), musée Vasili-Tchapaev (Čeboksary, 2004).
} 
Dans la première partie de l'article nous étudierons la reconversion des musées pendant la perestroïka et la création de nouveaux établissements, dans la deuxième partie nous verrons quelles nouvelles interprétations de l'histoire soviétique et de ces personnages illustres ont été proposées en réponse à la crise, enfin dans la troisième partie nous analyserons l'expérience des musées russes à la lumière de la discussion internationale sur la valorisation économique de la culture et sur la transformation des musées dans les pays occidentaux.

\section{LA RECONVERSION DES MUSEES PENDANT LA PERESTROIKA ET LA CREATION DE NOUVEAUX MUSEES}

\subsection{LA SITUATION DES MUSÉES IDÉOLOGIQUES EN URSS ET LE TOURNANT DE LA PERESTROÏKA}

En URSS, le fonctionnement des musées obéissait aux orientations générales de la politique culturelle de l'État (Gosselin, 1993). Cette dernière s'appuyait sur la création d'un réseau public d'établissements culturels accessibles à tous et l'instauration d'un système d'administration fortement centralisé (Sidorov et al., 1998).

La tradition soviétique muséale s'est formée dans les années 1920-1930. $\mathrm{Au}$ cours de la première décennie, les musées participèrent activement au mouvement de démocratisation de la culture, à l'ouverture des palais aux ouvriers et à la mémorialisation du mouvement révolutionnaire et des événements de la guerre civile. À partir des années 1930, toutes les institutions culturelles sont devenues propriété de l'État, leurs prestations étant incorporées aux plans quinquennaux. Pendant le premier et le deuxième plan quinquennal, les musées appuyèrent l'effort civilisateur de l'Etat qui s'efforçait d'éduquer les nouveaux habitants des villes et surtout les personnes nouvellement promues. Pendant la guerre, les collaborateurs des musées sauvèrent les collections. Au début des années 1950, les fonds des musées furent purgés lors de la Jdanovchtchina ce qui conduisit à la destruction de nombreux objets. Tel a été, par exemple, le destin tragique des fonds du musée de la Défense de Leningrad et du musée de la Révolution d'Octobre à Leningrad (Kantor, 2007).

La création du ministère de la Culture de l'URSS, en 1953, marque le début de la nouvelle période de stabilisation et de bureaucratisation de la gestion culturelle. Avec le Dégel, la mission idéologique des musées a pris de l'importance : l'État cherche désormais d'autres mesures que la terreur pour mobiliser la population. Dans les années 1970-1980, les musées furent pourvus de nouveaux moyens de conservation et d'exposition et obtinrent parfois de nouveaux bâtiments, modernes ou restaurés. En revanche, le 
contenu des expositions était sévèrement censuré ce qui incitait le personnel à contourner les prescriptions. Les musées d'histoire et de la littérature étaient contrôlés davantage que les autres établissements, en raison de la prépondérance de leur mission idéologique. Les expositions et les visites guidées devaient dissiper les doutes révisionnistes. Souvent, avant d'être nommés par le comité du parti de la région ou de la ville à la tête de ces musées, leurs directeurs avaient été responsables de l'idéologie dans les comités du parti. En revanche, le reste du corps des collaborateurs des musées était composé d'historiens professionnels, souvent détenteurs d'un titre de kandidat ou doktor nauk (docteur ou docteur d'État).

L'arrivée au pouvoir de Mikhaïl Gorbatchev fut marquée par une attention accrue à la culture et, plus généralement, aux affaires sociales, et par une augmentation du financement public destiné aux affaires culturelles. Les années 1986-1991 sont une période tout à fait particulière dans la vie des musées idéologiques. Ils ont vécu une crise de contenu, sans être confrontés encore aux problèmes économiques. C'est également une période de grande confusion au niveau des valeurs de la société, et du décalage, une fois de plus, entre les valeurs proclamées par le PCUS et les aspirations de l'intelligentsia. Tandis que Gorbatchev proclamait que les idées de Lénine étaient la source intellectuelle de la perestroïka (Gorbatchev, 1987), le public des musées n'était plus réceptif à l'idéologie communiste. La censure et le contrôle par le parti existaient encore théoriquement, tandis que la société bouillonnante exigeait la libéralisation idéologique immédiate de la culture. La loi de l'URSS sur la presse et les autres médias adoptée le 12 juin 1990 (Zakon SSSR ot 12 ijunja $1990 \mathrm{~g}$. «O pečati i drugikh sredstvakh massovoj informacii), qui abolissait la censure et instaurait la liberté de la presse et le libre fonctionnement des moyens d'information, a été adoptée en 1990 après un débat public animé.

En ces temps d'indécision où la société cultivée avait soif de libéralisme sans être sûre de l'obtenir, elle essayait d'anéantir les anciennes idoles. Les institutions idéologiques subissaient le mépris du public et craignaient de disparaître. Les quelques musées qui n'avaient pas de caractère mémoriel, ne possédant pas de fonds importants, ou n'ayant pu prouver leur utilité, furent définitivement fermés. D'autres institutions essayèrent de se reconvertir.

D'après la directrice de l'ancien musée de la Révolution d'Octobre, l'équipe du musée a été « contrainte » de fermer l'établissement en 1987, non pas à cause des directives de là-haut, mais pour d'autres raisons :

"L'année 1987 a été marquée par l'extrémisme. On condamnait, brisait et cassait tout ce qui symbolisait la période soviétique et, à plus forte raison, on attaquait la "grande » révolution d'Octobre. Pour sauver les objets et l'image du musée, on a juste laissé les salles mémorielles où travaillaient 
Lénine et les bolcheviks. Le reste de l'exposition étant, en effet, tendancieux, on l'a fermé et on s'est demandé ce qu'il fallait faire $»^{2}$.

Cette réflexion a pris plusieurs années et, en 1991, l'équipe du musée a dévoilé un nouveau concept de développement pour les cinq ans à venir. Depuis 1991, l'établissement a pris le nom de musée de l'Histoire politique de la Russie ${ }^{3}$.

En reouvrant son établissement sous un autre nom, l'équipe du musée avait décidé d'adopter une stratégie qui pourrait correspondre à l'époque de la transition. Les responsables estimaient que leurs visiteurs, qui traversaient une époque incertaine, s'intéresseraient à d'autres moments de crise politiques peu rares dans l'histoire de la Russie - les grandes réformes des années 1860, la révolution de 1905, la crise révolutionnaire de 1917, ou encore le dégel khrouchtchévien. En remplaçant la conception historique globale par des fragments, le musée cherchait des sujets qui pourraient toucher le public :

"On a décidé de montrer la Russie pendant les périodes de crises, et ne faire que des expositions temporaires et thématiques, en attendant que le savoir historique se stabilise. Par exemple, on a proposé l'exposition "Histoire du parlementarisme russe", consacrée à toutes les Doumas. On a fait également une exposition sur l'histoire du développement économique russe et la genèse de l'entreprenariat en Russie ${ }^{4}$.

Les musées de la littérature soviétique ont été confrontés aux mêmes problèmes, les grands maîtres du réalisme socialiste et les héros de leurs romans ayant été descendus de leur piédestal. La situation des musées Maxime-Gorki qui se trouvent à Moscou, à Nijni-Novgorod et à Kazan est, de ce point de vue, assez révélatrice. Nijni-Novgorod a été rebaptisée Gorki en 1936 et a porté le nom de l'écrivain, avant de retrouver son nom historique en 1991. La ville comptait plusieurs grands musées d'art et d'histoire, parmi lesquels le musée Gorki occupait une place importante. Celui-ci comportait trois sites historiques distincts : la Maison de Katchirine, ce grand-père méchant décrit dans Enfance, l'appartement du jeune Alekseï Pechkov et le musée littéraire, fondé par Gorki, dans sa ville natale. Pendant la perestroïka, le statut de cet ensemble commence à être mis en question, de même que le père du réalisme socialiste. Dans son entretien, la directrice du musée, en fonction depuis 1986, se souvient de la perestroïka comme d'un moment de transition très instructif, une période

2. Entretien de l'auteur avec Marta Potiforova, (ancienne) directrice du Musée d'Histoire politique de la Russie (Saint-Pétersbourg, 1999).

3. De même le musée central de la Révolution d'Octobre de Moscou qui fonctionnait depuis 1925 , s'est reconverti, pendant la perestroïka, en musée d'Histoire contemporaine de la Russie.

4. Entretien avec Marta Potiforova (1999). 
de vie intellectuelle intense où il fallait défendre le musée et défendre Maxime Gorki lui-même. Une vague de nouvelles publications de qualité variable a envahi l'espace public. En tant que professionnels du métier, les collaborateurs du musée devaient réagir aux falsifications de la biographie de l'écrivain et légitimer l'existence du musée dans cet environnement nouveau.

"Une série d'attitudes négatives contre Gorki nous a obligés à réagir. Et en nous mobilisant pour répondre, pour chercher des arguments, pour défendre notre position, nous nous sommes battus sur tous les fronts. En fait, Gorki n'avait pas besoin d'être défendu. Mais dans la mesure où nous en savions beaucoup sur lui, nous ne pouvions supporter certains avis erronés qui étaient publiés. Il fallait transmettre notre connaissance au public. De nouvelles sources apparaissant, nous avons beaucoup appris. On avait besoin de réinterpréter son image. Cette énergie négative nous nourrissait et s'est transformée en une autre énergie. Finalement, on a vécu ces années orageuses de manière très intense. Et maintenant que la vague s'est calmée, je commence à avoir peur de me retrouver dans une situation moins intense $\|^{5}$.

Les équipes des musées réorientent alors leurs expositions et les visites guidées en fonction des discussions publiques en cours au moment de la perestroïka. Plusieurs sujets nouveaux intéressaient à cette époque les visiteurs : l'histoire de la révolution d'Octobre vue sous l'angle d'un coup d'État ou d'un complot, l'histoire du Goulag et du système répressif soviétique, la vie de la société russe au début du $\mathrm{XX}^{\mathrm{e}}$ siècle, à l'époque du développement du capitalisme en Russie, la littérature, l'art et les personnages artistiques de l'Âge d'argent, l'émigration de l'entre-deux-guerres, la vie de la société soviétique entre la révolution et la Seconde Guerre mondiale, l'histoire des églises et du clergé.

Les entretiens avec des responsables des musées montrent qu'ils étaient confrontés à un défi, inexistant jusqu'alors : l'opinion des visiteurs et de la presse. Les relations avec le public deviennent leur principale préoccupation. Dans la période de l'après-guerre, les musées d'histoire soviétique accueillaient un très grand nombre de visiteurs, en raison des visites obligatoires des écoliers, des étudiants, des groupes touristiques organisés par des syndicats. Si, formellement, les musées étaient obligés d'attirer les visiteurs et de satisfaire leurs demandes, conformément à leur plan d'activités, dans la réalité l'opinion des visiteurs ne pouvait infléchir ni les thèmes des expositions, ni leur contenu. D'après le directeur du musée de l'Histoire de l'Armée et de la Marine de Saint-Pétersbourg, l'équipe du musée « avait pris l'habitude de travailler pour elle-même » (Korčagin, 1997, p. 74). L'apparition du public en tant qu'acteur important pouvant formuler et

5. Entretien avec Tamara Ryžova, directrice du Musée Maksim Gorkij à Nijni-Novgorod (2004). 
publier des opinions sur le musée a été vécue comme un changement crucial qui a nécessité de nouvelles compétences professionnelles.

Le tableau ci-dessous montre que le nombre des musées pendant et après la perestroïka a crû de façon continuelle à l'instar des objets dans les leurs fonds. Par contre, celui des visiteurs a considérablement régressé pendant cette période. C'est en 1992 - année de la «thérapie de choc » - que la fréquentation des musées a été la plus faible. Le problème des relations des musées avec leur public est désormais au centre des préoccupations des directeurs des établissements qui doivent reconsidérer leur rapport à la société.

TABLEAU 1

Quelques chiffres concernant les musées (1990-1994)

\begin{tabular}{|l|r|r|r|r|r|}
\hline & \multicolumn{1}{|c|}{1990} & \multicolumn{1}{|c|}{1991} & \multicolumn{1}{|c|}{1992} & 1993 & 1994 \\
\hline $\begin{array}{l}\text { Nombre de musées rattachés au } \\
\text { ministère de la Culture, dont: }\end{array}$ & 1257 & 1335 & 1379 & 1478 & 1602 \\
\hline - musées d'histoire & 270 & 273 & 278 & 317 & 323 \\
\hline - beaux-arts & 179 & 199 & 201 & 219 & 234 \\
\hline - étude et histoire de l'art & 19 & 28 & 29 & 32 & 35 \\
\hline - musées littéraires & 131 & 136 & 141 & 141 & 151 \\
\hline - architecture & 14 & 15 & 14 & 12 & 12 \\
\hline - sciences & 15 & 18 & 17 & 18 & 21 \\
\hline - techniques & 13 & 15 & 17 & 18 & 17 \\
\hline - musées thématiques & 12 & 18 & 12 & 13 & 12 \\
\hline - musées généralistes & 604 & 633 & 670 & 708 & 797 \\
\hline $\begin{array}{l}\text { Nombre d'objets dans le fonds } \\
\text { principal }\end{array}$ & 28700,2 & 35232,8 & 36787,2 & 37749,3 & 39294 \\
\hline Nombre de visiteurs (milliers) & 88959,2 & 80963,5 & 57648,2 & 64987,5 & 65312 \\
\hline
\end{tabular}

Source : Sidorov et al. (1998, p. 118).

Outre les visiteurs habituels (les familles, les groupes d'écoliers, les étudiants, les touristes russes) l'on voit désormais des touristes étrangers des pays occidentaux fréquenter les musées. Ces derniers constituent une catégorie de visiteurs tout à fait spécifique : à la différence des Russes qui se tournent de plus en plus vers la redécouverte de la Russie impériale ou de l'avant-garde artistique (Gaav \& Potapova, 1996), ce nouveau public est particulièrement avide d'information sur l'histoire soviétique certains veulent visiter des lieux mémoriels consacrés à la révolution d'Octobre avant même d'aller à l'Ermitage. Les directeurs des musées affirment que l'intérêt que les visiteurs étrangers ont manifesté pour leurs collections aurait été d'un grand soutien. Des collègues étrangers, spécialistes de la période soviétique, ou représentants des musées litté- 
raires et historiques des autres pays les ont également aidés, en insistant sur le fait que la période soviétique et ses personnages constituent un héritage original et utile pour la Russie nouvelle qui serait de nouveau valorisé dans quelques années.

L'isolement des institutions culturelles soviétiques n'avait, il est vrai, jamais été total, et certains musées bénéficiaient de contacts actifs avec des partenaires extérieurs à l'URSS. Pendant la perestroïka, les échanges internationaux se sont intensifiés, et les responsables et collaborateurs des musées les plus entreprenants en ont profité pour faire des stages dans les institutions étrangères. Les possibilités étaient multiples pour ceux qui souhaitaient en bénéficier et en avaient la possibilité. Les personnes qui ont commencé à établir des liens avec des collègues étrangers, à la fin des années 1980, sont devenues par la suite les acteurs principaux de la libéralisation économique de la culture en Russie (Tchouikina, 2010).

À la fin des années 1980, les associations indépendantes des musées ont émergé, à commencer par l'Union artistique des personnels des musées leningradois (Leningradskij tvorčeskij sojuz muzeinykh rabotnikov) en 1989. Des réseaux de musées, fondés sur le principe régional ou thématique, se sont multipliés dans les années 1990 et de nouveaux modèles de coopération ont vu le jour. En URSS, les relations professionnelles verticales étaient toujours privilégiées. Avec la libéralisation de la culture et de la vie politique, les relations horizontales entre les institutions partenaires se sont formées peu à peu. La réalité a évolué plus rapidement que la législation et les associations professionnelles ont contribué à la résolution des problèmes causés par le flou juridique. Dans le même temps, le prêt gratuit d'objets pour les expositions a commencé à se pratiquer.

\subsection{LES MUSÉES ET L'OBŠČESTVENNOST'}

L'obščestvennost', ou intelligentsia politiquement active, a soutenu ou, au contraire, dénigré les institutions culturelles existantes ; elle a milité en faveur de la création de nouveaux musées. Pendant que les anciens musées élaboraient des stratégies de reconversion et cherchaient des moyens pour justifier leur existence, de nouveaux musées ont vu le jour.

En 1989, Leningrad a fêté le centenaire de la naissance d'Anna Akhmatova. Des colloques, des soirées littéraires organisées dans des maisons de la culture, ont fait salle comble. Les invités étaient des intellectuels aussi bien soviétiques qu'émigrés. Ces derniers ont pu à cette occasion renouer avec Leningrad après des décennies d'absence. L'intelligentsia a suggéré de créer un musée dédié à Akhmatova dans sa ville natale. Le musée a vu le jour et s'est installé dans l'annexe du Palais Cheremetieff - Fontannyj dom où Akhmatova a passé presque toute sa vie dans un appartement communautaire. Les amis de la poétesse et leurs descendants ont apporté des objets 
mémoriels qui avaient été conservés dans des débarras. L'appartement d'Akhmatova est devenu, d'après sa directrice, « un musée de la poétesse et de sa génération ». De même, a été ouvert en 1992, le musée-appartement de l'écrivain satirique Mikhail Zochtchenko. Ainsi l'influence dévastatrice de la $\check{Z}$ danov̌̌čina, dont les traces se faisaient encore sentir tout au long de la période de stagnation, a été symboliquement vaincue.

À Nijni-Novgorod, l'obščestvennost' a exigé, à la même époque, la création d'un musée dédié à Andreï Sakharov, décédé en 1989. Le célèbre physicien et défenseur des droits de l'homme, qui avait été assigné à résidence à Gorki dans les années 1980, vivait sous surveillance dans un appartement austère. Un an après le décès de Sakharov, l'Institut de physique appliquée de l'Académie des sciences, la Société pour les droits de l'Homme et l'association « Mémorial » ont co-organisé un colloque pour lui rendre hommage. Les participants du colloque ont fait appel aux pouvoirs municipaux pour fonder le Musée Sakharov. Ce mouvement destiné à entretenir sa mémoire a coïncidé avec l'arrivée au pouvoir de Boris Nemtsov qui a soutenu le projet. En 1991, le musée a vu le jour.

La différence entre les nouveaux musées et ceux qui se sont reconvertis réside dans leur stratégie de développement. La reconversion des anciens musées soviétiques s'est organisée sur la base d'une réévaluation du potentiel de leurs fonds. Par exemple, le musée de la révolution d'Octobre de Leningrad possédait une grande collection de tracts et d'affiches de la période de la Première Guerre mondiale et de la Guerre civile : des trains de propagande circulaient sur les fronts avec pour mission de conserver soigneusement, pour les générations futures, tous les documents y compris ceux provenant des camps ennemis. Par la suite, le musée a continué de collectionner toute la production écrite relevant de la vie politique. Sur la base de ces fonds, interdits à l'exposition depuis les années 1930, le musée s'est transformé en 1991 en musée de l'Histoire politique de la Russie.

De nouvelles structures, tels que les musées-appartements d'Akhmatova et de Zochtchenko ou encore celui de Sakharov, se trouvent dans une situation différente car, au départ, leur fonds était maigre. Après un bref succès au moment de leur ouverture, ces établissements ont été délaissés par le public. Cela a incité leurs directeurs à élargir leur domaine d'activité en organisant des soirées littéraires et des rencontres artistiques, en proposant des visites guidées de haut niveau. Par exemple, le musée Sakharov organise des conférences sur l'histoire de la physique pour les adolescents et des visites guidées en anglais sur l'histoire du mouvement des droits de l'homme en URSS. L'exposition est présentée en deux langues, en russe et en anglais, en raison de sa fréquentation pour moitié par les étrangers.

Les musées du $\mathrm{XX}^{\mathrm{e}}$ siècle, autant ceux qui se sont reconvertis que les nouveaux, attirent des forces politiques diverses, dont le spectre va des 
libéraux-démocrates aux communistes, qui veulent associer ces lieux symboliquement forts à leurs stratégies. Cette tendance, déjà visible à la fin des années 1980, s'est intensifiée après 1991, avec la création de nouveaux partis politiques. L'année 1991 devient le grand tournant qui voit le bouillonnement de l'obščestvennost' se transformer en une véritable lutte d'influences politiques. Si la critique du système commence comme une tentative d'améliorer le socialisme, après le putsch et l'abolition de la domination idéologique du PCUS, l'intelligentsia se divise par rapport au changement survenu. Des lieux de commémoration de l'époque soviétique deviennent, dès lors, des lieux de batailles historiques, surtout pour les partisans du régime communiste.

La directrice du musée Maxime-Gorki de Nijni-Novgorod se souvient qu'au moment du référendum sur le retour du nom historique de la ville, en 1991, certaines associations ainsi que des particuliers, loin d'être indifférents, faisaient montre d'une attention particulière et bienveillante à l'égard du musée qui recevait des dons de la part de bienfaiteurs anonymes.

"Un jour, alors qu'on fêtait l'anniversaire de Gorki, nous avons organisé un colloque et devions recevoir ses descendants, j'ai découvert dans le hall du musée plusieurs caisses de vodka "Gorkovskaïa ». Des sponsors inconnus nous les avaient laissées en cadeau. En outre, on nous apportait toujours du champagne le jour de son anniversaire, surtout à la Maison Katchirine. Dans ces gestes, on voyait que certains citadins n'étaient pas d'accord avec ce qu'ils entendaient sur Gorki, et ils voulaient nous soutenir. Même si l'attitude dominante était plutôt négative, on voyait une autre attitude, qui venait d'en bas. Cela était très intéressant et stimulant pour nous $»^{6}$.

Quant au Musée Gorki à Kazan, il est devenu dans un contexte de montée de la conscience nationale tatare, un lieu à la fois de soutien de la culture russe et de rencontre des deux cultures.

\section{LA REDECOUVERTE DE L'HÉRITAGE LOCAL ET L'ÉCLATEMENT DES INTERPRETATIONS GLOBALES}

À l'époque soviétique, les musées d'histoire et de la littérature du $\mathrm{XX}^{\mathrm{e}}$ siècle étaient censés proposer une interprétation globale de leur exposition. Toutes les vitrines et tous les objets convergeaient vers une interprétation déjà familière au visiteur. D'un point de vue idéologique, la visite devait confirmer les idées reçues, plutôt que d'en proposer de plus originales. Les musées dans toutes les régions de Russie étaient obligés de proposer les mêmes interprétations qu'à Moscou. Des metodički et instrukcii (conseils

6. Entretien avec Tamara Ryjova, directrice du musée Maxime-Gorki à Nijni-Novgorod (2004). 
méthodologiques et instructions) de toutes sortes avaient été rédigés pour indiquer la façon adéquate de représenter les choses. Par exemple, dans les nombreux musées Lénine il fallait toujours montrer les mêmes traits du caractère d'Ilitch : sa simplicité et sa proximité du peuple, son goût de la lecture, son esprit anti-bourgeois, son obsession du travail. Les particularités locales étaient, dans une grande mesure, gommées de l'interprétation globale. Pendant la perestroïka, on observe deux tendances inverses : la redécouverte de l'héritage local et l'éclatement des interprétations globales et universelles au profit de l'exposition d'éléments disparates.

\subsection{LIER LES MUSÉES À LA VILLE, METTRE EN VALEUR L'HÉRITAGE URBAIN}

Après 1987 et surtout après 1991, l'universalisme de la période soviétique cède la place au localisme et au particularisme de l'époque libérale. Avec la décentralisation politique et culturelle croissante, le fonctionnement des musées et leur développement sont davantage influencés par les spécificités locales ; leur stratégie s'inscrit de plus en plus dans la politique des municipalités. Les pouvoirs municipaux, en quête d'une niche économique pour la ville afin d'améliorer son image, essaient de développer les institutions culturelles, malgré le manque de ressources financières. L'architecture et la planification urbaine soviétique étant sévèrement critiquées, les villes s'efforcent de s'embellir. Des publications consacrées à l'histoire des rues, des places, des bâtiments, des jardins, et des mystères des villes se multiplient. De nouveaux monuments commémorent les exploits de certains citadins contemporains de l'époque impériale. Comme le remarque à juste titre Georges Nivat, la Russie est frappée, à cette époque, d'une « hypermnésie », en compensation de l'amnésie des décennies antérieures (Nivat, 2007).

Les musées d'histoire et de la littérature du $\mathrm{XX}^{\mathrm{e}}$ siècle ont suivi le mouvement général de la redécouverte de l'histoire locale. Depuis la perestroïka, tous les musées sont devenus très attentifs à leurs locaux. Ils conçoivent des expositions consacrées à l'histoire de leurs bâtiments et mettent en valeur l'esprit du lieu. On souligne l'originalité architecturale du bâtiment ou de l'immeuble, son rôle dans l'histoire du quartier et de la ville en général.

D'après la directrice du musée Anna-Akhmatova à Fontannyj Dom, dans l'interprétation que fait le musée de l'œuvre de la poétesse et de son impact sur sa génération, son lieu d'habitation - Fontannyj Dom a quasiment autant d'importance que la personnalité mythique d'Anna Akhmatova. Le message principal de l'exposition est de présenter Anna Akhmatova comme une poétesse pétersbourgeoise, incarnant la continuité culturelle. Le point de vue stéréotypé et idéologiquement soutenu dans le discours officiel soviétique et postsoviétique voit l'année 1917 comme le début d'une rupture historique et culturelle. L'effort créateur d'Akhmatova 
proposait de voir la culture russe dans une perspective plus large. Le fait d'avoir vécu à proximité du palais soutenait cet esprit de continuité.

"Le palais et le jardin justifient à $90 \%$ notre concept. Akhmatova a vécu à peu près 35 ans ici, de 1917 à 1952. C'est un musée de la culture pétersbourgeoise, c'est le seul lieu d'habitation d'un poète du vingtième siècle qui ait été si intimement lié à la culture du palais, à l'ancienne culture noble, traditionnelle et familiale. Akhmatova héritait de cette culture, était en contact avec elle $»^{7}$.

En associant la vie singulière de la poétesse (qui était en même temps typique de l'intelligentsia de l'époque) à l'histoire d'un lieu ancien, sublime et mystérieux, le musée entend répondre au désir de chacun d'inscrire son histoire personnelle et familiale dans l'histoire longue. En outre, il tente d'établir une continuité entre la culture de l'Âge d'argent et celle, non officielle, de la période soviétique.

Le lien entre la fin du XIX ${ }^{\mathrm{e}}$ siècle et la fin du $\mathrm{XX}^{\mathrm{e}}$ devient un sujet de discussion à tous les niveaux de la société qui a besoin de repères. L'inscription de l'histoire de Leningrad dans un contexte noble pétersbourgeois, la cohabitation, parfois peu justifiée, de la révolution avec la tradition, est une stratégie caractéristique des musées d'histoire du $\mathrm{XX}^{\mathrm{e}}$ siècle qui sont nombreux à occuper d'anciens palais.

Le palais Roumiantsev, situé au 44, Quai des Anglais, au bord de la Neva abrite l'une des filiales du Musée de l'Histoire de Saint-Pétersbourg, consacrée aux années 1920-1940. Après avoir emprunté des couloirs et des escaliers anciens, traversé quelques salles dédiées à la famille Roumiantsev et à l'histoire du bâtiment, le visiteur se plonge subitement dans l'ambiance d'une ville soviétique, avec les reconstitutions des cuisines des appartements communautaires, des crèches pour les enfants des ouvriers, des abris antiaériens, des obus et des uniformes de la période de la guerre. À l'exposition sur l'histoire de la guerre ouverte depuis de nombreuses années, se sont ajoutées deux autres salles dans les années 1990, l'une retraçant la NEP et l'autre la reconstruction socialiste pour répondre au grand intérêt du public pour la vie quotidienne de cette époque. La présentation de l'histoire du bâtiment et de la famille des propriétaires est également un ajout récent.

Le Musée de l'histoire politique de la Russie occupe un hôtel particulier qui avait appartenu dans les années 1910 à la célèbre danseuse de ballet Kchesinskaïa, par ailleurs maîtresse d'un des grands-ducs. Après l'émigration opportune de la danseuse au début de l'année 1917, l'hôtel particulier fut occupé par l'état major du parti bolchevik. Lénine faisait des discours du balcon de ce bâtiment. Outre les expositions dédiées à l'histoire poli-

7. Interview avec Nina Popova, directrice du musée Anna-Akhmatova (2001). 
tique et économique de la Russie, une partie du musée est consacrée à l'histoire de ce bâtiment. Plusieurs pièces relatent la vie de Kchesinskaïa et de sa famille, d'autres pièces - les activités de l'État major des bolcheviks. L'histoire du bâtiment est aussi contradictoire que l'histoire politique de la Russie. Chef-d'œuvre de l'architecture « art nouveau » russe du début du siècle, lieu artistique et politique, cet hôtel particulier symbolise, lui aussi, le retour à la culture de l'Âge d'argent. Il incarne un lien spirituel entre le monde de l'émigration parisienne et l'intelligentsia russe d'aujourd'hui et témoigne de la volonté manifestée par les nouvelles élites d'occuper les lieux jadis habités par les anciennes.

Une stratégie semblable a été adoptée par la direction du musée Smolny, créé en 1991, héritier d'un Musée Lénine. L'ancien Institut des Jeunes filles nobles fut, lui aussi, occupé en automne 1917 par les bolcheviks avant de devenir l'État major du coup d'État d'Octobre puis la résidence du premier gouvernement soviétique. Le premier cabinet et le logement de fonction de Lénine en tant que chef de l'État se sont également trouvés dans ce bâtiment jusqu'en mars 1918. Depuis la reconversion du musée, il met en scène à la fois la culture de la noblesse et la culture révolutionnaire et soviétique. La plus grande partie de l'exposition est consacrée désormais au fonctionnement du célèbre Institut des Jeunes filles nobles. Une autre partie montre le cabinet de travail de Lénine, sa chambre et laisse entrevoir la vie de ce bâtiment avant le déménagement du gouvernement à Moscou. Enfin, une troisième partie du musée est consacrée aux activités de Smolny en tant que centre administratif de la ville pendant le blocus de Leningrad.

Auparavant, l'histoire des bâtiments et de leurs propriétaires historiques ne faisait jamais partie de l'exposition d'un musée. L'héritage de la noblesse n'était pas mis en valeur. Aujourd'hui, la vie privée des anciens propriétaires des beaux lieux est devenue un objet d'intérêt.

\subsection{UN ÊTRE HUMAIN CONFRONTÉ À DES CIRCONSTANCES EXTRAORDINAIRES}

L'abandon de l'universalisme soviétique ne concerne pas uniquement les lieux et les bâtiments. C'est également pendant la période de la perestroïka que s'est dessiné un retour à l'être humain avec ses préoccupations privées, ses besoins, ses peurs, sa grandeur et sa petitesse, sa capacité de simple mortel d'infléchir le cours de la grande histoire, de résister aux difficultés, de s'opposer au régime politique.

Les musées ont commencé à proposer des expositions diverses sur le thème de l'être humain confronté à des circonstances extraordinaires. Ce questionnement a encore gagné en popularité dans les années 1990 avec la crise économique. La société tout entière avait besoin de reconsidérer, non seulement l'histoire politique, mais encore sa mémoire collective. Les 
musées essayaient de contribuer à ce mouvement, et d'aider la population à libérer la mémoire collective des interdictions et des modèles imposés par l'histoire soviétique tendancieuse.

Dans le questionnement du public, l'expérience de la survie au Goulag était au premier plan des épreuves difficiles et tragiques de l'époque soviétique. Les aspects linguistiques, juridiques, culturels, psychologiques et psychiatriques de cette expérience faisaient l'objet de vives discussions (Jurgenson \& Anstett, 2009). Le mouvement pour la liquidation des taches blanches de l'histoire, et le mouvement pour la réhabilitation (y compris politique) des victimes battait son plein (Ferretti,1989). Des petits ou grands musées du Goulag ont commencé à se former, dès la fin des années 1980, sur les lieux des camps, à commencer par l'île des Solovki, où le premier camp pour les prisonniers politiques avait été fondé en 1923. Les musées d'histoire soviétique centraux, ceux de Moscou et de Saint-Pétersbourg, ont également essayé de traiter également ce sujet, en mettant l'accent sur la mémoire familiale de l'expérience carcérale et sur l'impact du phénomène du Goulag sur la société urbaine soviétique. Des objets légués aux musées par la population (des objets d'art fabriqués dans les camps, des jouets improvisés, des photos) ont trouvé leur place dans les vitrines. Alors que sur l'emplacement des camps on commémorait l'histoire du lieu, dans les musées centraux des villes capitales on essayait de montrer le retentissement que l'expérience carcérale avait eu dans la vie privée des familles, et comment la détention avait été vécue (Anstett, 2009).

Même les musées de la Seconde Guerre mondiale, moins touchés par les changements idéologiques, ont suivi cette nouvelle tendance à interpréter l'expérience humaine dans des situations hors du commun. Ainsi, le responsable du musée de la Défense de Leningrad considère le Blocus de la ville comme une période où les limites des possibilités humaines ont été poussées à l'extrême.

"L'attitude était différente. L'un ne résistait pas et mourait, l'autre arrivait à s'en sortir. Cette expérience de survie a eu une importance colossale. Non seulement pour notre pays. Pour le monde entier. Pour savoir de quoi un être humain est capable! »"

Les musées historiques et littéraires consacrés aux personnages célèbres se sont mis à explorer la rencontre d'un être humain avec des grandes idées. Comment celles-ci sont-elles interprétées par l'homme ? Comment changent-elles son destin ? Ce motif est transversal dans le musée-appartement d'Andreï Sakharov à Nijni-Novgorod. Le musée mémoriel réinterprète la pensée scientifique du physicien et ses idées sur les droits de l'homme du point de vue de l'époque contemporaine.

8. Interview avec Valeri David, vice-directeur du musée de la Défense de Leningrad (2000). 
Dans les années 1990, un autre aspect sera ajouté à ce questionnement : la possibilité d'ascension sociale pendant les périodes de crises, la contribution des self-made men à la culture de la société en transition. Ce sujet est traité dans les musées dédiés à Maxime Gorki. Selon la directrice d'un de ces musées, l'écrivain peut intéresser les visiteurs en tant qu'homme russe qui s'est construit tout seul. Non seulement il a pu sortir de la misère et devenir un intellectuel de grande envergure, mais il a également influencé le contexte politique de son époque et créé de nouveaux genres littéraires. Sa personnalité permet également de sonder l'éternel problème de la relation ambiguë d'un Russe à l'Europe et aux États-Unis et les sentiments d'un intellectuel russe vivant en Occident.

\subsection{LA DOMESTICATION DES RÉVOLUTIONNAIRES ET L'ABANDON DE L'IDÉOLOGIE}

L'abandon des interprétations totalisantes, au profit de la valorisation de l'expérience humaine, a abouti à un résultat inespéré dans le cas des musées-appartements des révolutionnaires. Des personnages, jusqu'alors mythologisés par l'idéologie soviétique, redeviennent des êtres humains simples et communs. Leurs objets personnels, anciennement interdits à l'exposition, trouvent leur place légitime dans les intérieurs réels.

Les musées de Vladimir Ilitch Oulianov (Lénine) sont particulièrement intéressants à cet égard. La divinisation de sa personne et le culte de sa personnalité dans la culture politique de l'URSS (Tumarkin, 1983; Brossat, 1990) et dans les musées en particulier (Welikanowa, 1993) ont été supplantés par la présentation de traits ordinaires (et quelconques) du leader de la révolution. Lénine est descendu brusquement de son piédestal et s'est rapproché de ses contemporains.

Jusqu'au milieu des années 1980, on soulignait son esprit anti-bourgeois. D'après l'interprétation canonique soviétique, ce grand homme était indifférent au confort de la vie matérielle, se contentant du strict minimum. Certains de ses objets personnels étaient, d'ailleurs, interdits à l'exposition car ils pouvaient être jugés petit-bourgeois, telle une couverture de lit en dentelle blanche. Depuis la perestroïka, ces objets personnels, au contraire, jouent un rôle de premier plan, faisant de l'ombre à la personnalité politique du stratège et à l'originalité de ses idées.

Depuis le début des années 1990, Saint-Pétersbourg compte six musées consacrés entièrement, ou partiellement, à Lénine, commémorant ses lieux d'habitation ou de travail ${ }^{9}$. Les entretiens avec les responsables de

9. Il s'agit de l'actuel musée Raznočinnyi Peterburg (Le Saint-Pétersbourg des roturiers), l'ancien musée de l'Histoire du mouvement révolutionnaire (1992-2006) qui était auparavant un musée Lénine ; l'appartement appartenant à son gendre Mark Elizarov dans lequel le leader des bolcheviks habita peu avant la révolution de 1917 ; l'appartement du révolu- 
ses endroits mémoriels ainsi que les publications dans la presse montrent l'intention d'humaniser le personnage de Lénine et de diminuer la portée de ses idées. Pendant les visites guidées, la spécificité de sa pensée politique, son concept de parti professionnel ou sa position au sein de la deuxième Internationale ne sont que très rarement évoqués. Ses lieux d'habitation ont été transformés en musées de la vie quotidienne de la fin du $\mathrm{XIX}^{\mathrm{e}}$-début du $\mathrm{XX}^{\mathrm{e}}$ siècle. Conformément à cette nouvelle mission des musées, la personnalité et les intentions du révolutionnaire perdent en importance ; ce sont ses traits particuliers, ceux d'un représentant de toute une génération, qui sont mis en avant.

La responsable du musée-appartement de Mark Elizarov, le gendre de Lénine, a formulé la vocation de son musée de la manière suivante

"Grâce au musée Lénine qui n’avait jamais été fermé, notre ville a pu garder un appartement unique en son genre, le seul qui expose si amplement la vie quotidienne de l'intelligentsia du début du siècle. Nous ne faisons aucune propagande, nous ne transmettons aucun message idéologique. Nous vous montrons ce qui a réellement existé. Nous n'interprétons rien. Nous exposons les activités des personnes qui habitaient cet appartement les Oulianov, les Elizarov, et Vladimir Ilitch, aussi, bien sûr. Évidemment, nous exposons les événements de 1917, d'avril à juin 1917, quand Lénine résidait ici. Plus tard, nous prévoyons d'ajouter une salle sur l'histoire de cet arrondissement, du district Petrogradskji. Parce que c'est ici que la ville est née » ${ }^{10}$.

Un autre ancien musée Lénine (un appartement où le jeune Oulianov louait une pièce en 1894-1895 avant son exil sibérien) a été reconverti, en 1992, en musée d'Histoire du mouvement révolutionnairedémocratique ${ }^{11}$, et a choisi une stratégie de présentation très semblable à celle du musée-appartement précédemment décrit.

"Évidemment, après l'écroulement de l'idéologie ancienne et du culte de Lénine au début des années 1990, le musée ne pouvait pas rester comme avant. Son charme consiste maintenant à montrer l'histoire de la vie quo-

tionnaire Allilouev, le gendre de Staline, où Lénine passait de temps en temps ; le musée « Šalaš » (la hutte) qui se trouve dans un faubourg, non loin de la ville où Lénine se cacha en 1917 après l'interdiction du parti bolchevik ; son lieu de travail dans l'État-major du parti bolchevik en 1917, dans l'hôtel particulier de Kchesinskaïa qui avait été réquisitionné ; et enfin, son cabinet de travail, dans le bâtiment de l'Institut de jeunes filles nobles «Smolny », utilisé pendant et après le coup d'État réussi.

10. Interview avec Nelly Privalenko, directrice du musée-appartement des Elizarov (2000). 11. Depuis qu'il est devenu le Saint-Pétersbourg des roturiers (cf. note 9), outre ses expositions toujours consacrées à V.I. Oulianov, ce musée entend explorer la vie des quartiers « laids », ceux qui se trouvent « derrière la Fontanka », des lieux de résidence des étudiants pauvres et d'autres pétersbourgeois solitaires (voir à ce sujet le site du musée : http://www. museum.ru/m175). 
tidienne des Pétersbourgeois à la charnière des XIX et XXe siècles. La chambre de Lénine, qui appartenait à l'ancien musée, s'est idéalement insérée dans le nouveau concept du musée et dans sa nouvelle exposition. Parce qu'on peut la regarder dans une perspective nouvelle: on voit ici, tel quel, le logement typique d'un «locataire du coin» (Glezerov, 2001).

Le musée Smolny a également changé l'aspect du cabinet de Lénine en le remplissant avec des objets mémoriels qui montrent le côté «normal » du personnage:

"Bien évidemment, nous avons préservé le bureau de Lénine, mais nous l'avons garni d'objets plus humains. Par exemple, nous avons mis sa casquette. Nous avons mis le chapeau de Nadejda Konstantinovna. Vladimir Ilitch a acheté cette casquette en 1917, en route vers la Russie, c'est une casquette française. Nous avons aussi mis leur parapluie. Nous avons ainsi ajouté des éléments qui ont humanisé ce cabinet. Et cela intéresse sans aucun doute les visiteurs. Nous avons mis les meubles qui se trouvaient dans son bureau à l'origine, et qui avaient été enlevés plus tard. Parce qu'auparavant on arrangeait tous les musées Lénine de manière identique. Maintenant on montre comment c'était en réalité. On montre tout ce qui se passait réellement. C'est pour ça que j'ai dit dès le début que nous ne transmettions aucun message idéologique $»^{12}$.

Les lieux chargés d'idéologie à l'époque soviétique devaient avant tout confirmer une bonne version de l'histoire. Indépendamment de leur profil, tous les musées véhiculaient les mêmes idées. Aujourd'hui, l'on assiste à l'éclatement de cet ensemble - fragmenté en une multitude d'éléments tirés de la vie quotidienne, de quelques histoires particulières, histoires des bâtiments et des quartiers. L'ampleur internationale ou globale des activités révolutionnaires ou communistes a été réduite pour laisser la place à la description de ce qui s'est passé dans ces lieux. On peut avoir l'impression que Lénine est important avant tout comme propriétaire d'objets, comme résident d'un quartier et représentant de son groupe social. Les musées d'histoire et de la littérature soviétiques fonctionnent ainsi depuis la perestroïka comme un prolongement de la mémoire collective des citadins. La question de la place de l'histoire officielle et de l'historiographie nouvelle dans leur travail reste à élucider.

12. Interview avec Vera Tioutcheva, directrice du musée (2000). Ces citations reflètent la recherche d'une nouvelle perspective pour les musées Lénine, mais aussi la méfiance des responsables à l'égard des journalistes et de toute autre personne censée décrire leurs activités. La fin des années 1980 et le début des années 1990 ont été marquées par une vague d'hostilité et de mépris pour ces établissements «idéologiques ». À la fin des années 1990-début des années 2000, au moment où les entretiens ont été conduits, les responsables des institutions étaient encore sur la défensive. 
Pour des raisons politiques, la révision critique de l'ensemble de la période soviétique et de son historiographie n'a pas été faite par les historiens russes. Les musées fonctionnent dans un contexte où le savoir historique est en recomposition. Dès la fin des années 1980, les historiens et les amateurs d'histoire, après avoir rejeté la version canonique soviétique, ont découvert de nombreux travaux d'historiens occidentaux. L'école totalitaire a gagné du terrain (Shearer, 1998, pp. 568-569) et le terme de totalitarisme a commencé à être très amplement utilisé dans l'espace postsoviétique. Un certain nombre d'ouvrages américains, relevant surtout de cette tradition historique, a été assez rapidement traduit en russe. Par la suite, avec l'ouverture des archives et la création de nouvelles maisons d'éditions, de nombreuses recherches historiques de toutes tendances (aussi bien «critiques » que « patriotiques») ont été publiées, sans pour autant que se dégage une réelle vue d'ensemble. L'on constate que la situation supposée temporaire au début des années 1990 a duré presque deux décennies : à une vision de l'histoire totalisante a succédé une vision éclatée. Pourtant, un seul de nos interlocuteurs - le responsable du musée de la Défense de Leningrad - a évoqué l'absence de vision historique cohérente comme un obstacle au travail des musées. Il semble que depuis la perestroïka, les musées d'histoire et de la littérature soviétique ont pour principale vocation de compléter la mémoire familiale et culturelle, sans expliquer ce qui s'est passé.

De ce point de vue, la situation en Russie est différente de celle des autres pays de l'Europe de l'Est. Dans ces pays, les musées d'histoire, à la veille des événements de 1989, attendaient des directives. Les visiteurs s'ennuyaient ou s'indignaient en regardant les anciennes vitrines ou en écoutant les interprétations historiques dépassées (Soulé, 1990 ; Simionescu \& Padiou, 1990). En revanche, dans les années 1990 et surtout 2000, les historiens et le public ont commencé à réviser et à réinterpréter le passé communiste plus intensément que leurs homologues russes, pour s'en défaire et se lier de plus en plus à l'histoire de l'Europe de l'Ouest (Mink \& Bonnard, 2010). Les établissements nouvellement fondés comme La Maison de la Terreur à Budapest (Losonczy, 2006 ; Rev, 2008) ont fait revivre les horreurs des répressions. Les musées est-européens ont participé assez activement à l'interprétation et souvent à la réprobation de ce passé, tandis qu'en Russie la question de la responsabilité dans les répressions ainsi que celle des compromis politiques et identitaires de l'époque soviétique restent, dans une grande mesure, refoulés (Ferretti, 1995 ; Gessat-Anstett, 2007). Lorsque des jugements définitifs sont émis, c'est généralement le fait d'associations. Avec l'Église, ce sont elles qui, par exemple, s'occupent en Russie de la commémoration des lieux d'exécution et d'inhumation de masse (Jemkova, 2009 ; Rousselet, 2007 et 2008). En 
somme, la fonction principale des musées d'histoire en Russie postsoviétique n'est pas de problématiser le passé mais d'apaiser la société.

\section{La liberalisation de la CUlture et la PROFESSION MUSEale APRes 1991}

Les années de la perestroïka ont abouti à un autre changement significatif dans la vie des musées, à savoir la mise en question du modèle de la gestion culturelle soviétique, et l'introduction des idées occidentales relatives à la libéralisation de la sphère culturelle. Déjà, au cours de la seconde moitié des années 1980, des réformes économiques avaient été entreprises dans le secteur culturel afin d'offrir plus d'autonomie aux établissements. Mais la tendance libérale dans le domaine de la culture a été renforcée par une nouvelle politique économique dans le pays, moins favorable aux institutions culturelles publiques que la précédente. Après 1991, les coupures de budgets, de plus en plus fréquentes, ont forcé les musées à chercher des ressources supplémentaires. Cependant, la nouvelle crise, bien que «budgétaire », ne peut pas être observée uniquement sous l'angle économique, car les solutions anti-crise qui ont été proposées ont véhiculé une certaine idée de la vocation des musées et de la culture dans son ensemble. Et, plus que l'arrivée de la glasnost', c'est cette dernière quesion qui a divisé la communauté muséale.

Les nouveaux principes législatifs ont été formulés en 1992, avec l'adoption de la Loi fondamentale de la Fédération de Russie relative à la culture. Les principes du fédéralisme ont été appliqués dans le domaine de la culture comme ailleurs. L'autonomie culturelle des minorités ethniques a été garantie, les principes de la liberté artistique, de la liberté de création des institutions culturelles privées et des associations ont été affirmés. Dans le même temps, le système de l'administration culturelle n'a pas été transformé de façon radicale, il est resté hiérarchique et fortement centralisé. Le ministère de la Culture de la Russie a, en effet, conservé son rôle centralisateur sur fond de décentralisation politique et économique. Néanmoins les régions ont depuis lors une plus grande responsabilité dans la gestion culturelle et les responsables des établissements ont une plus grande liberté d'action.

Pour comprendre l'évolution des musées russes depuis 1991, il convient de prendre en compte l'état de la profession muséale dans les autres pays. Dans quel contexte international ces établissements évoluaient-ils quand le rideau de fer est tombé ? En sciences sociales, les publications consacrées à la situation des musées en Europe, au Canada et aux USA, font ressortir que, depuis le début des années 1980, ces institutions étaient en train d'adopter un nouveau modèle de fonctionnement (Ballé \& Poulot, 2004 ; Jaumain, 2000 ; Rolland \& Muravskaya, 2008). En effet, les musées 
dans les pays occidentaux entrent alors dans une phase de transformation, inspirée par une nouvelle politique culturelle qui s'est traduite par le tournant vers le «capitalisme culturel » (Jameson, 1991), la dissolution de la haute culture dans un «tout » culturel (Cusset, 2008) et un abaissement relatif du statut de cette dernière. D'après de nombreux essais et travaux de chercheurs issus de différentes disciplines et tendances politiques, la culture perd à cette époque sa vocation critique, et acquiert en revanche une dimension économique jamais connue jusqu'alors (Jameson, 1991 ; Boltanski \& Chiapello, 1999 ; Cusset, 2008 ; Florida, 2005 ; Hall \& Landry, 1998). Cette valorisation économique de la culture stimule le développement des villes et la décentralisation de la gestion culturelle. La tendance à la décentralisation est aussi bien typique de la Grande Bretagne dans le cadre des réformes néolibérales de M. Thatcher que de la France, avec la politique culturelle du gouvernement socialiste. Les musées deviennent des acteurs centraux de la politique culturelle des villes et jouent un rôle central dans la compétition que celles-ci entretiennent entre elles : leur nombre augmente considérablement. Une unité territoriale, même très petite, souhaite avoir son musée pour pouvoir se construire en tant que communauté. La démocratisation et la capacité d'accueillir un grand nombre de visiteurs devient un défi pour les musées occidentaux qui commencent à élaborer des programmes ciblés pour atteindre les strates les plus diverses de la population. D'après certains conservateurs désespérés, les musées, jadis orientés vers les visiteurs cultivés, connaisseurs de l'art et de l'histoire, sont aujourd'hui au service de l'industrie touristique et sont envahis par des foules de « barbares » (Clair, 2007 ; 2011).

La Russie, pendant et après la perestroïka, a voulu suivre l'exemple des pays occidentaux, et même si les voix critiques se sont faites entendre, la tendance dominante, soutenue par le public cultivé et par les médias, a été à l'abandon progressif de la tradition économique et gestionnaire de l'URSS. Les responsables des institutions culturelles n'ont pas échappé à cette tendance générale dans leur intention de suivre les modèles étrangers. Les fondations caritatives qui ont entamé leurs activités en Russie au début des années 1990 ont grandement contribué à la transmission du savoir étranger sur le sol russe. La Fondation Soros a ouvert plusieurs filiales à Moscou, Saint-Pétersbourg, Novossibirsk, Ekaterinbourg. Vers le milieu des années 1990, la culture est devenue une des directions stratégiques de ses activités. Et parmi les institutions culturelles russes financées par la Fondation Soros, ce sont les musées qui sont déclarés prioritaires (Muzei v epokhu peremen, 2001). D'après les entretiens avec les directeurs des musées, certains d'entre eux ont commencé à participer aux séminaires, colloques et programmes éducatifs organisés avec le soutien financier de la Fondation Soros dès 1991. L'Institut pour les études culturelles de Moscou (Institut kulturologii), avec notamment son Laboratoire 
d'ingénierie des musées (www.futuremuseum.ru), est devenu le médiateur principal entre les experts étrangers et les responsables des musées dans les régions. L'exemple des musées britanniques pendant les réformes de M. Thatcher a souvent été évoqué comme modèle à suivre pour la Russie.

Quelles ont été les idées véhiculées par la doctrine de la libéralisation de la culture ? D'un point de vue théorique, il s'agissait de passer du positivisme de l'époque industrielle au post-modernisme de l'ère de l'information. Cette approche postmoderne préconisait de travailler avec beaucoup d'ironie et de distance; d'intégrer des éléments ludiques ; d'utiliser des messages, des images ou des citations inattendues et provocatrices, en mélangeant des faits historiques avec des éléments du temps présent. Il était également conseillé d'établir des partenariats avec d'autres musées ou, éventuellement, d'autres institutions culturelles, afin de produire des événements novateurs. De nouveaux moyens techniques, permettant aux visiteurs d'appuyer sur des boutons et de consulter des informations sur des écrans, étaient considérés comme indispensables dans les institutions postmodernes. Des programmes interactifs de toutes sortes devaient diversifier l'offre. S'agissant des nouveaux savoirs et compétences professionnelles à acquérir, les responsables des musées devaient apprendre à écrire des projets, pratiquer le marketing, les relations publiques, le planning stratégique, l'informatisation, le travail avec les fondations et avec les sponsors.

Vers la fin des années 1990, le discours sur la modernisation des musées en Russie ressemblait beaucoup à ce qu'on peut lire sur ce sujet dans les autres pays. Mais si le libéralisme culturelétait très présent dans la réflexion, on le remarquait moins sur le terrain. Certes, les plus grands musées, d'importance fédérale, se sont rapidement transformés : ils ont changé la structure de leurs départements, en construisant des restaurants et des magasins à l'intérieur de leurs bâtiments, ouvert des filiales à l'étranger et stimulé l'innovation et les collaborations (Chekova, 2004). En revanche, les établissements modestes, surtout dans les villes petites et moyennes, ne donnent pas l'impression d'être des temples des musées postmodernes et témoignent d'une continuité culturelle et historique étonnante.

Des inégalités et des différences frappantes distinguent, aujourd'hui encore, les établissements et leurs dirigeants. Si certains responsables des musées ont participé aux séminaires et programmes de formation continue depuis 1991, en Russie ou à l'étranger, d'autres n'ont jamais été sollicités pour participer à de tels programmes, et n'ont jamais pris l'initiative de participer à des concours de projets organisés par la Fondation Soros (Tchouikina, 2010). La modernisation des musées a été partielle et n'a pas touché de manière égale toutes les régions et tous les établissements (Čuvilova, 2009). Le système de la gestion culturelle au niveau central est resté quasiment inchangé et les possibilités d'établir des partenariats indé- 
pendants restent très éphémères. Dans les années 2000, les responsables des établissements confiaient, dans leurs entretiens, que leur objectif principal était d'établir de bonnes relations avec leurs supérieurs hiérarchiques dans les départements de la culture. La verticale du pouvoir, héritée de l'époque soviétique, et renforcée par la suite, reste très solide dans ce secteur. Comme le renouvellement des élites dans les régions n'a pas été significatif, et que les horizons culturels de ces élites politiques restent limités par leur appartenance générationnelle, il n'est pas surprenant que la modernisation des musées, entamée au début des années 1990, n'ait pas été probante partout.

Néanmoins, le tournant vers le libéralisme culturel, annoncé en 1992, a eu une portée réelle en ce qui concerne le discours des spécialistes, l'orientation générale du développement et les efforts que font le ministère de la culture et certaines municipalités. Il n'y a pas de doute que les musées russes suivent la même voie que les musées européens.

\section{Conclusion}

La période de la perestroïka a profondément marqué les trajectoires professionnelles des responsables des musées d'histoire et de la littérature soviétiques. Ils ont obtenu une assez grande autonomie d'expression, se sont libérés du joug de la censure et ont reconstruit leur rapport à la société, en travaillant autrement avec les visiteurs et la presse.

Loin d'être vécus de manière semblable dans toute la Russie, les changements politiques et économiques ont touché les institutions culturelles de manière inégale. Ces institutions ont joué un rôle différent selon l'importance des villes, leurs ressources financières et leur composition ethnique. Cette diversité représente le plus grand changement par rapport à l'époque soviétique. Dans l'après-guerre, les institutions culturelles fonctionnaient en effet de manière identique dans tout le pays, remplissant le plan des prestations culturelles et transmettant les mêmes idées. Mais pendant la perestroïka, la personnalité des dirigeants des institutions a joué un rôle déterminant. Les uns voulaient maintenir la tradition, les autres, plus ouverts à l'innovation et à l'expérimentation, ont décidé de donner une nouvelle orientation à leurs établissements.

Les années 1990 ont vu l'apparition de fondations caritatives, devenues des acteurs importants, qui ont soutenu des projets innovants dans certains musées et ont stimulé des partenariats. Cependant, le système de subordination administrative et de financement des activités par les instances supérieures n'a pas été modifié. Les musées sont restés entièrement 
dépendants, comme à la fin de l'URSS, soit directement du ministère de la Culture, soit des comités de la culture auprès des municipalités. Les initiatives individuelles des dirigeants comptent beaucoup, mais ils sont confrontés à de nombreux obstacles d'ordre économique et politique.

La transmission du savoir sur l'époque soviétique, dont l'héritage reste très controversé, s'effectue de manière neutre, en évitant toute position critique. Les musées ont réussi à construire de bonnes relations avec leur public fidèle qui participe aux diverses manifestations culturelles diverses organisées par ces institutions. Par contre, le rapport des musées avec l'histoire et les historiens reste indéterminé. Ceux-ci ont adopté, pendant la perestroika, une stratégie défensive, refusant la mission idéologique et évitant de mettre en valeur le contenu des idées ; ils ont continué à travailler de cette manière au cours de ces vingt dernières années. Ayant abandonné une vision cohérente de la construction du socialisme dans un seul pays, ils proposent désormais une vision éclatée, un puzzle d'éléments non assemblés, qui relèvent de la vie privée tant des hommes illustres que des simples mortels, sous un régime dont on évite de définir le sens.

\section{BIBLIOGRAPHIE}

AnstetT Elisabeth (2009), «Un musée pour une anamnèse. De l'usage des œuvres d'art dans une muséographie de la Terreur », in Elisabeth Anstett \& Luba Jurgenson (dir.), Le Goulag en héritage. Pour une anthropologie de la trace, Paris : Petra, pp. 143-155.

Ballé Catherine \& Poulot Dominique, eds. (2004), Musées en Europe : Une mutation inachevée, Paris : La documentation française.

Boltanski Luc \& Chiapello Eve (1999), Le nouvel esprit du capitalisme, Paris : Gallimard.

Brossat Alain (1990), «Le culte de Lénine : le mausolée et les statues », in A. Brossat, S. Combe, J.-Y. Potel, J.-C. Szurek, À l'Est, la mémoire retrouvée, Paris : La Découverte, pp. 165-197.

Cheкоva Ekaterina (2004), "Organizational structure of Russian State Museums: Recent Innovations", International Journal of Arts Management, Vol.6, $\mathrm{n}^{\circ}$ 2, pp. 44-53.

Clair Jean (2007), Malaise dans les musées, Paris : Flammarion.

Clair Jean (2011), L'hiver de la culture, Paris : Flammarion.

Cusset Yves (2008), La décennie : le grand cauchemar des années 1980, Paris : La Découverte.

Čuvilova I.V. (2009), Istoričeskie ekspozicii regionalnykh muzeev v postsocialističeskij period, Sankt-Peterburg: Aleteia. 
Ferretti Maria (1989), «La réhabilitation de Boukharine: les enjeux de l'histoire en Union soviétique », Batailles de mémoires : Histoire et mémoire en URSS et en Europe de l'Est, publié par le Groupe de recherche Mémoire grise à l'Est, Paris : BDIC, IMSECO, pp. 79-93.

Ferretti Maria (1995), «La mémoire refoulée. La Russie devant le passé stalinien », Annales. Histoire, Sciences Sociales, vol. 50, n 6, pp. 1237-1257.

FLORIDA Richard (2005), Cities and the Creative Class, London; NY: Routledge.

Gessat-Anstett Elisabeth (2007), Une Atlantide russe : Anthropologie de la mémoire en Russie post-soviétique, Paris : La Découverte.

Glezerov S. (2001), « Tipičnyj uglovoj žilec Vladimir Ulianov » (L'habitant typique du coin, Vladimir Oulianov), Nevskoe vremia, 01.02.

Gorbatchev Mikhail (1987), Perestroïka : vues neuves sur notre pays et le monde, Paris : Flammarion.

Gosselin Aurélie (1993), La politique des musées russes, 1917-1991, Paris : Découvrir.

Hall Peter \& Landry Charles (1998), Des villes innovatrices et durables, Loughlinstown : Fondation européenne pour l'amélioration des conditions de vie et de travail ; Luxembourg : Office des publications officielles des Communautés européennes.

JAMESOn F. (1991), Postmodernism, or the Cultural Logic of Late Capitalism. Durham: Duke University Press.

Jaumain S., ed. (2000), Les musées en mouvement. Nouvelles interprétations, nouveaux publics. Bruxelles : Université Libre de Bruxelles.

JEMkova Elena (2009), « Les répressions staliniennes à Moscou et les lieux d'inhumation de masse ", in Elisabeth Anstett et Luba Jurgenson, Le Goulag en héritage. Pour une anthropologie de la trace. Paris: Petra, 2009, pp. $115-129$.

Jurgenson Luba \& Anstett Elisabeth (2009), « Héritages et mémoires du Goulag : Pour une anthropologie de la trace, in Elisabeth Anstett et Luba Jurgenson, dir., Le Goulag en héritage. Pour une anthropologie de la trace. Paris : Petra, pp. 11-17.

KANTOR Ioulia (2007), «Le musée de l'Histoire politique à Saint-Pétersbourg », in Georges Nivat, dir., Les sites de la mémoire russe. Tome 1. Géographie de la mémoire russe, Paris: Fayard, pp. 200-207.

KorČAGIN Evgenij (1997), « Magazin pri muzee » (Les boutiques de musées), in A.D. Margolis, dir., Muzei v period peremen (Les musées à l'époque des transformations), Sankt-Peterburg: Kontrfors, pp. 74-75.

Losonczy Anne-Marie (2006), « La muséification du passé récent en Hongrie post-communiste: deux mises en spectacle de la mémoire », Revue d'Études Comparatives Est-Ouest, vol. 37, n 3, pp. 97-112. 
Mink Georges \& Bonnard Pascal (2010), Le Passé au présent: Gisements mémoriels et actions historicisantes en Europe centrale et orientale, Paris: Michel Houdiard.

Muzei v epokhu peremen (Les musées à l'époque des transformations) (2001), Institut «Otkrytoe obščestvo » (Fond Sorosa Russia) v podderzhku rossiiskih museev, 1996-2000, Moskva: OGI.

Nivat Georges (2007), « Mémoire russe, oubli russe », in Georges Nivat (dir.), Les sites de la mémoire russe. Tome 1. Géographie de la mémoire russe, Paris : Fayard, pp. 9-59.

Oushakine Sergueï (2009), The Patriotism of Despair: Nation, War and Loss in Russia, Ithaca: Cornell University Press.

Rev Istvan (2008), "The Terror of the House », in Robin Ostow, (Re)visualizing National History. Museums and National Identities in Europe in the New Millennium, Toronto: University of Toronto Press, pp. 47-89.

Rolland Anne-Solène \& Muravskaya Hanna, dir. (2008), De nouveaux modèles de musées? Formes et enjeux de créations et rénovation de musées en Europe XIX - XXI siècles, Paris : L'Harmattan.

Rousselet Kathy (2008), «Les mémoires de la Grande Terreur : Boutovo », in Marie-Claude Maurel \& Françoise Mayer (dir.), L'Europe et ses représentations du passé, Paris: L'Harmattan, pp. 131-145.

Rousselet Kathy (2007), « Butovo : la création d'un lieu de pèlerinages sur une terre de massacres », Politix, $\mathrm{n}^{\circ}$ 77, avril, pp. 55-78.

SHEARER David (1998), «From divided consensus to creative disorder: Soviet history in Britain and North America ", Cahiers du monde russe : Russie, Empire russe, Union sovietique, États indépendants, vol. 39, ${ }^{\circ}$ 4, pp. 559-591.

Sidorov Evgueni et al. (1998), « Rapport national sur la politique culturelle de la Fédération de Russie présenté par le ministère de la Culture de la Fédération de Russie », La Politique culturelle de la Fédération de Russie, Strasbourg : Éditions du Conseil de l'Europe, pp. 9-206.

Smith Kathleen (2002), Mythmaking in the New Russia: Politics and Memory During the Yeltsin Era, Ithaca; London: Cornell University Press.

Simionescu Paul \& Hubert Padiou (1990), « Roumanie: comment le musée national de Bucarest racontait l'histoire ", in A. Brossat, S. Combe, J.-Y. Potel, J.-C. Szurek, À l'Est, la mémoire retrouvée, Paris : La Découverte, pp. 212-228.

Soulé Véronique (1990), «Le musée de la police à Prague », in A. Brossat, S. Combe, J.-Y Potel., J.-C. Szurek, À l'Est, la mémoire retrouvée, Paris : La Découverte, pp. 198-211.

Tchouikina Sofia (2010), “The Crisis in Russian Cultural Management: Western Influences and The Formation of New Professional Identities in the 1990s-2000s", The Journal of Arts Management, Law and Society, Vol.40, $\mathrm{n}^{\circ} 1$, pp. 76-91. 
Tumarkin Nina (1983), Lenin lives! The Lenin Cult in Soviet Russia, Cambridge: Harvard University Press.

WeliKanowa Olga (1993), "Der Lenin-Kult in sowjetischen Muséen », Osteuropa, Heft 10, 1993, pp. 929-938. 